\title{
Presentation, treatment and natural course of severe symptoms of urinary tract infections measured by a smartphone app.
}

Akke Vellinga, Karen Farrell, Roisin Fallon, Daniel Hare, Una Sutton-Fitzpatrick, Martin Cormican

BMC Medical Research Methodology

Akke Vellinga

School of Medicine and Irish Primary Care Clinical Trials Network

National University of Ireland, Galway

Galway

Ireland

Akke.vellinga@nuigalway.ie

Karen Farrell

Discipline of General Practice

HRB Primary Care Clinical Trials Network Ireland

National University of Ireland, Galway

Farrellk93@gmail.com

Roisin Fallon

Discipline of General Practice

HRB Primary Care Clinical Trials Network Ireland

National University of Ireland, Galway

roisinfallon95@gmail.com

Daniel Hare

Department of Clinical Microbiology

University Hospital Galway

Galway

Ireland

Danielhare5000@gmail.com

Una Sutton-Fitzpatrick

Specialist Registrar in Clinical Microbiology

Tallaght University Hospital 
Dublin

Ireland

suttonfu@tcd.ie

Martin Cormican

National Clinical Lead AMRIC (Antimicrobial Resistance and Infection Control Team) Health Service Executive (HSE)

Discipline of Bacteriology

National University of Ireland, Galway

Martin.cormican@hse.ie

Corresponding author: Akke Vellinga, akke.vellinga@nuigalway.ie 


\begin{abstract}
Background

Urinary tract infections (UTI) are one of the most common conditions in females. Current information on presentation, management and natural course of the infection is based on paper diaries filled out by patients and subsequently posted. The aim of the study was to explore the feasibility of a smartphone app to assess the natural course and management of urinary tract infections.
\end{abstract}

\title{
Methods
}

A smartphone app was developed to collect data from study participants presenting in general practice with symptoms of a UTI. After initial demographic and treatment information, symptom severity was recorded by the patient upon a reminder on their smartphone which occurred twice daily for a period of 7 days or until symptom resolution.

\section{Results}

A total of 181 women aged 18-76 downloaded the smartphone app. Duration of symptoms was obtained from the results of 178 participants. All patients had a urine sample submitted, most patients were prescribed an antibiotic (90\%) and $39 \%$ had a positive culture. Moderately bad or worse symptoms lasted a mean of 3.8 days (median 4 ) and $70 \%$ of patients indicated that they were cured on day 4 after consultation. This compares with other research assessing symptom duration and management of UTIs where data is collected by paper diaries. Patients were very positive about the usability of the smartphone app and often found the reminders supportive. Based on the feedback and the analysis of the data, some suggestions for improvement were made.

\section{Conclusions}

Smartphone diaries for symptom scores over the course of infections are an efficient and acceptable means to collect data in research. 


\section{Keywords:}

1. Urinary tract infections

2. General Practice

3. Smartphone application 


\section{Background}

Urinary tract infections are one of the most common conditions female patients consult their GP with (1). More than half of women consult their GP with a UTI at least once in their lives $(2,3)$.

UTIs are considered self-limiting infections $(4,5)$ but generally antibiotics are prescribed empirically (6-8). Prescription of antimicrobials for UTI account for a substantial number of prescriptions in primary care contributing to increased risk of adverse drug effects, burden of resistant infections (9, 10) as well as increased costs (11). The duration of more severe symptoms in women presenting in primary care with suspected UTI which turned out to be susceptible to the antibiotic prescribed was 3.3 days on average (2). However, the duration increased to 4.7 for those with resistant infections and 4.3 days for women with symptoms but no significant bacterial growth (urethral syndrome) (2). An international comparison of presentation and management of UTI in four European countries, found wide differences in management, with antibiotic prescribing for $59 \%-95 \%$ of the women presenting, but no differences in the time to resolve moderately bad to worse symptoms, which took a median of 4 days (12).

All studies reporting on the management of UTI base their findings on the use of paper diaries, which are provided to women at the moment of consultation and requested to be returned by post after symptoms resolve $(5,13-15)$. Such diaries are also used in UTI trials in which antibiotic treatment is compared with symptomatic treatment for UTI in general practice $(16,17)$.

In one observational study to record the natural course of UTIs $64 \%$ of the symptom diaries were recovered (2) and another observational cohort over 4 countries $70 \%$ of the diaries were recovered (18). A Danish study observing the effect of point of care testing for UTI recovered $85 \%$ of the patient diaries (19). All of these studies have used additional resources to contact patients and urge them to post their diaries.

Compliance with paper diaries has been compared to electronic diary keeping for patients recording pain three time daily for 21 days. The difference between electronic and paper recording was analysed and showed a high level of feigned compliance in the use of paper diaries (20). Different versions of electronic diaries have been piloted and tested in various environments to record symptoms in for instance inflammatory bowel disease (21), overactive bladder (22), and asthma (23).

Smartphone apps are increasingly used in care innovation research and provide new opportunities to develop interventions (24). In pain settings, apps have been most widely used to record diary entries both in observational (25) and clinical trial settings (26) but applications are also implemented for other conditions (27-29). Smartphone diaries have been shown to be highly efficient and perform generally better than paper based diaries.

In our own SIMPle study we embedded an observational study to follow patients with UTI using a smartphone app (30). In this feasibility study 71 patients showed that a smartphone app was well received and that once a patient committed to reporting their symptoms, they would continue doing so for the duration of the study (31).

\section{Methods}

Aim

To describe the presentation and course of suspected UTI in females presenting in primary care and document outcome of urine culture, antibiotic resistance of bacterial growth as well as antibiotic prescribing using a smartphone app with reminders for data collection . 


\section{Design}

Observational feasibility study of women presenting with symptoms of a UTI.

\section{Setting and participants}

Between November 2018 and December 2019, six general practices in the west or Ireland enrolled female adult patients with symptoms of a UTI and a smartphone. After obtaining consent, the patient received a patient number which was texted to a central number, to record the patient's phone number, and a link to download the app was returned. Both patient and practice received a payment for each download of the smartphone app.

\section{Follow up}

A telephone call was be made to all patients on day 4 and day 28 (or as soon as possible after this day). The aim of the day 4 phone call was to confirm the symptom score. Additionally, information on adverse events, experience using the app and return consultation with a GP were recorded.

\section{Smartphone app}

A smartphone app was developed to collect data from study participants (figure 1). At enrolment, patients were requested to record demographic information (age, recent UTI, marital status, children, medical card) as well as treatment details (antibiotic treatment yes/no, name of antibiotic, duration).

To record severity of symptoms patients received a reminder twice a day, once during the morning at 9 (or 10 and 11 AM if no entry was made) and once in the afternoon at 6 (or 7 and 8 PM if no entry was made). Patients were reminded to provide their symptom score for seven days and, if no recovery occurred, up to 14 days or until symptom recovery. Symptom recovery was defined as a score of less than 2 (moderate) for each of the symptoms.

Symptom severity included four symptoms; dysuria (painful/burning urination), frequency, urgency and low abdominal pain. Symptoms were scored from 0 (not at all) to 4 (as bad as it gets). An additional question 'Do you feel cured' was included after the symptom score to allow comparison with a Scandinavian trial in which this was the main outcome score (32).

On day 0 and day 5 , patients were also asked to fill out a UTI-related questionnaire on impairment in daily live-activities on the Activity Impairment Assessment (AIA) (33) as well as self-rated health in general and on the particular day (on a visual analogue scale from 0-100) (34). The AIA is a 5-item questionnaire to assess the amount of time an individual's work or regular activities has been affected as a result of their UTI. Patients respond to the AIA items on a 5-point Likert scale with the response options 'none of the time', a little of the time', 'some of the time', most of the time' and 'all of the time'. The questions are: Did you have to cut down on time at work, could you accomplish less, were you limited in the kind of work you did, did you have difficulty performing work, did your UTI interfere with social activity.

\section{Sample size}

Considering a mean difference of half a day clinically significant, a sample of 92 patients $(\alpha=0.05$ and $\beta=0.2$ ) should detect such a difference in the mean duration of symptoms (score moderate or worse on the four items (dysuria, frequency, urgency and low abdominal pain) (5)) of 3.8 (standard deviation 3.0) on day 4.

\section{Data analysis}

The duration of symptoms includes the day the patient consulted their GP. Mean duration of symptoms was based on the day their mean total symptom score was moderately bad or worse and similarly for the duration of each symptom separately. Mean duration to cure is based on a yes answer 
to the question 'Do you feel cured?'. Symptom resolution is based on the day all symptoms are 0 . As the app was filled out for a duration of 7 days, return of symptoms within this 7 days was included in the duration of symptoms. End of symptoms was also indicated on the first day when a patient ceased making any more entries.

Antibiotic prescribing was recorded on the day of the consultation, and patients were asked every time if they were still taking the antibiotic. The antibiotic prescribed was assessed retrospectively in the context of the microbiology culture result to assess if the organism tested as susceptible to the antibiotic chosen empirically.

Urethral syndrome was defined as symptoms of suspected UTI but with no significant bacterial growth detected on culture (2).

Analysis was performed using STAT 13.0 as well as IBM SPSS version 26. Tableau was used to visualise the data.

\section{Laboratory analysis}

Urine samples were collected for each patient participating. Samples were send to the Microbiological lab of the University Hospital Galway for analysis. A positive sample was based on a colony count of $>10,000 \mathrm{cfu} / \mathrm{ml}$ pure growth observed after overnight incubation on chromogenic agar. Mixed growth was also recorded and included for further analysis. Organisms detected in pure culture were identified by MALDI-ToF (Bruker). Susceptibility testing was performed using EUCAST disc diffusion methodology and interpretive criteria. 


\section{Results}

A total of 181 patients downloaded the smartphone app. Their mean age was 29.7 years, (SD 14, median 22) with a range between 18 and 76 . Of the women, $28 \%$ were married or in a relationship and $69 \%$ did not have children, $18 \%$ had one or two children and $13 \%$ had 3 or more children. More than one third (37\%) of the patients were entitled to free medical and GP care, while $63 \%$ had to pay for their GP visit as a private patient. Of the participants, $66 \%$ had or were doing a university degree and $34 \%$ had a secondary education. A total of $30 \%$ were working, $6 \%$ were home makers and $54 \%$ were students.

Of the 181 patients, 3 never filled out any symptom score, while all others provided at least one symptom score. On day 4, 39 did not provide a symptom score, but 13 of them provided one on the previous day.

Of the 181 patients, $163(90 \%)$ were prescribed an antibiotic. Nitrofurantoin was the most often prescribed $(66 \%)$ followed by trimethoprim (12\%), the others being co-amoxyclav (7\%), amoxicillin $(5 \%)$, fosfomycin (3\%) and a quinolone (1\%).

The question which was asked at every entry, if they took their antibiotic or if they used any pain medication, was filled out irregularly and could not be analysed.

\section{Symptom duration}

Symptoms started on average 5.6 (SD 6.3) days before they consulted their GP with a median of 4 days (Interquartile range 5). Duration of symptoms after they consulted their GP was 4.2 days (3.0) and time to cure (indicated by the patient) was 3.8 (SD 3.2) days. Table 1 and figure 2 show in more detail the mean duration of moderately bad or worse symptoms. The most common symptom rated moderately bad or worse on the first day was urgency (64\%), followed by frequency (62\%), lower abdominal pain (55\%) and dysuria (40\%) and the duration of each symptom is shown in figure 3 . There were no significant differences between types of UTI or whether an antibiotic was prescribed or not . An overview of the percentage of patients who indicate that they felt cured each day from consultation, is presented in figure 4 . Overall, $70 \%$ indicated that they felt cured on the fourth day after consultation (day 5 ) and this $72 \%$ amongst those who had an antibiotic prescribed.

Overall, $90 \%$ had moderate to worse dysuria for 4 days or less, $85 \%$ frequency, $81 \%$ urgency and $67 \%$ pain in lower abdomen. Overall, symptoms lasted 4 days or less after seeing their doctor for $57 \%$ of patients (figure 4).

\section{Microbiology}

Overall, $39 \%$ or 70 patients had a positive culture. Enterobacteriaceae (mainly E.coli) were cultured from 50 patients ( $31 \%$ of all patients) followed by S. saprophyticus from 10 patients (6\%). A total of 32 $(18 \%)$ were mixed cultures and $22(12 \%)<10,000$ cfu per ml. Resistance to at least one of the tested antibiotics was recorded in 33 of the $54(61 \%)$ isolates for which this could be assessed. Comparing the antibiotic prescribed with the isolate and its resistance, showed that 36 (55\%) out of the 66 antibiotics tested as susceptible against to the antimicrobial prescribed while for $27(41 \%)$ this was not known. For 3 patients the organism cultured tested resistant to the antibiotic prescribed. One of these patients was contacted to change the antibiotic (from co-amoxyclav to nitrofurantoin).

\section{AIA and general health}

Little difference was observed for the AIA score; the mean AIA score on 0 was 6.6 (SD 5.0) and on day 5 this dropped to 4.1 (SD4.6). A paired t-test of patient providing a score on both days showed significance $(p<0.05)$ Patients were asked to rate their general health on day 0 (mean 75.6, SD 17.3) 
and day 5 (76.3, SD 18.2) (figure 5). Their health on the day was rated at 55.8 (SD 20.1) on day 0 and 71.9 (SD 19.0) on day 5. The difference between their rated health on day 0 and day 5 , as well as the difference with overall health was significant. On day 5 , their health score was still significantly different from the overall health score, but the overall health score on day 0 and day 5 were not statistically different.

The total symptom score on day 0 was correlated with the AIA $\left(R^{2}=23 \%\right)$ and the health score $(17 \%)$ on day 0 and similarly for day 5 .

\section{Associations}

There was no difference in duration of symptoms, total symptom score on day of consultation, AIA and health status on day of consultation between those who did and did not go to university, or between students and non-students. Only total symptom score was significantly higher for those not in a relationship compared to participants who were married or with partner but no other differences were observed. Age was not correlated with any outcome (duration, total score, days with symptoms before consultation, health score or AIA).

\section{Day 4 and day 28 phone call}

A call was answered by 152 participants on day 4 and 128 on day 28 .

On day 4, 71 or $40 \%$ indicated they felt cured, which compares to $72 \%$ according to the app data. Comparison showed that $69 \%$ (103) indicated the same on the call as they did on the app, $6(4 \%)$ indicated cured over the phone but not on the app and 41 (27\%) say they were cured in the app but not when asked over the phone. The total symptom score on day 4 according to the phone call was 3.8 days (+/-3.5) according to the call (152) and 2.6 days (+/-2.6) according to the app (142).

When asked about side effects, $29 \%$ (44) reported side effects from the medication. The main side effects recorded were stomach upset/diarrhoea (17) and thrush (4) while 18 did not further specify any side effects and 5 reported urine discoloration (all patients reporting they had received nitrofurantoin).

Of the patients who answered a call on day $28,32(25 \%)$ consulted the GP again for symptoms of UTI during the 28 days since initial consultation. Patients who consulted their GP had significantly $(p=0.006)$ higher symptom duration $(5.7(4.3)$ versus $3.9(2.6))$ but no difference in symptom score on the day they consulted. There was no difference in antibiotic or painkiller prescribing or which antibiotic was prescribed between those who did and did not reconsult. Patients who reconsulted were significantly older (35.9 (SD17.8) versus 27.9 (SD 12.4). On day 5, the AIA score (6.9 versus 2.9) was significantly higher and the health score (59.7 versus 75.1 ) was significantly lower for those who reconsulted.

\section{Evaluation of app}

For the day 4 call 116 patients provided further comments on their UTI and/or the use of the app while 128 on day 28 . In general, comments were positive. Some patients liked the fact that the app reminded them of their medication and improvement of their symptoms.

'Handy for reminding in morning and evening to take my medication and think about my symptoms more and if they were improving or not' 
'App was good as made me check in with myself, showed me how it was a recovery process'

'App was good, not time consuming, interesting for herself to track day to day feelings'

Overall, the app was received well:

'App was good, quick and easy to use'

Even though a few comments were made in relation to the repetition on the questions (2).

'Felt like they were the same questions over and over again' 


\section{Discussion}

It was shown that the use of a smartphone app to track patient's symptoms of UTI is an efficient and effective approach to replace paper diaries. Our estimates of the mean and median duration of symptoms from the date of consultation was 4.2 days and 4 days respectively. This is comparable with the observations made in the studies of natural course of infection according to previous papers in which paper diaries were used $(2,12)$. However, the mean and median duration of individual symptoms were rated lower in the app compared to the paper diaries from both studies. Comparing our results, in particular when prescribed an antibiotic, to the arm in the trials showing antibiotic prescribing, shows a mean duration of moderate to worse symptoms at 4.6 days in the trial (16) compared to 4.3 in our study. The 'do you feel cured', as the main outcome in the Scandinavian trial was also included in our study, $74 \%$ indicated cured on day 4 after the consultation when taking the antibiotic, while in our study, $72 \%$ indicated cured in the group where an antibiotic was prescribed. In general, outcomes are similar to the outcomes from other studies. The main difference and challenge in comparing different studies and trials is the day on which outcomes are measured, i.e. whether this includes the day of consultation or not. Inclusion of the method of measuring in a core outcome set for UTI should be clearly defined (35).

UTIs are considered self-limiting infections $(4,5)$ but generally antibiotics are prescribed empirically (6-8). Prescription of antimicrobials for UTI accounts for a substantial number of prescriptions in primary care contributing to increased burden of resistant infections $(9,10)$ as well as increased costs (11). Our study showed that $90 \%$ of the patients presenting were prescribed an antibiotic of whom $81 \%$ received a firstline antibiotic (8). Only $33 \%$ of patients had bacteriological confirmation of their UTI. Very similar figures of prescribing are confirmed in Wales, England and Spain, while the Netherlands show much lower prescribing (59\%), however confirmed UTI was around $17 \%$ in Wales and England, $42 \%$ in Spain and $64 \%$ in the Netherlands. Even though more literature is emerging suggesting that symptomatic treatment of UTI is often sufficient and acceptable $((16,17)$, most GPs are hesitant doing so. Compared to 10 years previously, GPs are prescribing more in line with guidelines, as in 2010 in a study conducted in the same region only $26 \%$ prescribed a firstline antibiotic (7). The efforts made since to decrease the use of co-amoxyclav in general practice and encourage the use of (firstline) prescribing guidelines, seem to be having an effect $(30,36)$.

Our overall AIA score on the day of the consultation was 6.6 and 4.1 on day 4, compared to the trial of Gagyor et al. in which respectively 8.9 and 1.1 was recorded. It is not clear why such large differences were observed. The validation of the AIA score showed a total score on the day of consultation of 7.6, which still is higher than our finding, but they do not provide a follow up score (37). The inclusion of the health score may measure general wellbeing on the day of the consultation better, and shows high concordance between the general health score on the day of the consultation and day 4 . This general health score may provide a better insight in the severity of the UTI and its impact on wellbeing.

The day 4 phone call was included to see how the smartphone scores compare with the answer provided over the phone and $40 \%$ indicate to feel cured while $72 \%$ indicated this to be so on their smartphone. The total symptom score according to the app was also lower than according to the phone call. Again, it is not clear why people report lower scores on the app compared to a phone call.

During the follow-up period, $25 \%$ reported a reconsultation and $27 \%$ reported side effects of some sort, which is in line with the reported reconsulations in the four country study.

In relation to the use of a smartphone app to collect data and considering the comments as well as the analysis of the data, a number of conclusions can be made: 
- A smartphone app is an efficient and effective tool to collect real-time data in research on infections

- Our app asked for twice daily scores, which was decided to capture differences between morning and afternoon scores. Many did not fill out the app twice a day and those who did, did not show major differences between morning and afternoon. It also introduced an additional step in the analysis, using the mean score for the day, or if only one score was provided, to use this score.

- Patients are interested and committed to use their app at the start of the study. At this stage a few additional questions could be included that will improve its use.

- The app should request at the start what time of the day is most convenient to fill out the app. This should then be the trigger for the reminder on the phone to fill out their app.

- Type and duration of antibiotic prescribed: On the day after their course is intended to finish, patients can be asked if they have any medication left. This would avoid repeatedly asking if they took their antibiotic.

- For painkiller use, this should only be included if part of particular interest to the main research question.

\section{Strengths and limitation}

To our knowledge, this is the first prospective study to describe the natural course of a (urinary tract) infection using a smartphone app. Smartphone apps are frequently used for chronic conditions, where patients have more time and are not in distress when asked to enrol in a study. The acceptability and ease of use of the smartphone app has been shown for both patient use and completeness of data. However, participants may have been selectively rather than randomly invited to participate but no reliable logs of potentially eligible patients were kept. We did encourage GPs to enrol any age group and the range in age from 18-76 years shows clearly that age was not a limitation to participate in our study. More likely restrictions to enrolment are expected to be due to the additional time necessary to consent patients to the study, in particular as UTI consultation are usually considered straightforward and short.

A few questions arise with the results obtained, in particular in relation to discrepancies between phone call scores and smartphone scores from the same day. Other research into the course of infections using a smartphone app may be performed in the future which may shed more light on this. However, no other study was identified using smartphones for infection symptom scores, so our findings could not be confirmed. However, the similarities of our outcomes compared to studies with paper diaries is encouraging. In particular, for trials, where consent will be required anyway, smartphone diaries may be considered in the future, which could significantly reduce energy and time spend to collect outcomes after a patient leaves the practice.

\section{Conclusion}

Smartphone diaries for symptom scores over the course of infections are an efficient and acceptable means to collect data in research. 


\section{Declarations}

\section{Ethical approval}

Ethical approval was obtained from the Irish College of General Practitioners. Individual informed consent was obtained from every participant before enrolment in the study. Consent had to be confirmed at the registration when downloading the smartphone app.

\section{Consent for publication}

Not applicable

\section{Availability of data and materials}

Access anonymised patients data can be obtained from the corresponding author upon request. Code for the smartphone app can be obtained from the corresponding author and used freely if referenced appropriately.

\section{Competing interest}

None of the authors declared any competing interest.

\section{Funding}

Funding was obtained as part of the SATIN study of the Irish Primary Care Clinical Trials Award from the Health Research Board, Ireland (CTN-2014-011).

\section{Authors' contributions}

AV designed, managed and supervised the study, performed the statistical analyses and interpretation, and wrote the manuscript. KF and RF organised GP and patient enrolment, collection of smartphone data and the follow up phone calls. DH and USF provided the microbiological information. MC provided support during the design of the study, helped to interpret the results, and corrected the manuscript. All authors read drafts of the manuscript and approved the final manuscript.

\section{Acknowledgement}

This study was set up after the early discontinuation of the SATIN trial. We like to thank the members of the SATIN steering committee and the Clinical Research Facility Galway for their input in the early development of the smartphone app.

We would like to thank the GP practices and patients who took part in our study. 
Table 1: Mean, median and (standard deviation) of symptoms according to UTI type and antibiotic prescription.

\begin{tabular}{|c|c|c|c|c|c|c|c|c|}
\hline & & Symptoms & Dysuria & Frequency & Urgency & $\begin{array}{l}\text { Lower } \\
\text { abdominal } \\
\text { pain }\end{array}$ & Resolution & Cured \\
\hline $\begin{array}{l}\text { Overall } \\
\text { duration, } \\
\text { Mean }\end{array}$ & 178 & $4.2(3.0)$ & $\begin{array}{l}2.0 \\
(2.6)\end{array}$ & $2.6(2.6)$ & $3.0(2.9)$ & $2.9(3.3)$ & $\begin{array}{l}10.7 \\
(20.9)\end{array}$ & $\begin{array}{l}3.8 \\
(3.2)\end{array}$ \\
\hline Median & & 4 & 1 & 3 & 2 & 2 & 6 & 3 \\
\hline \multicolumn{9}{|l|}{ UTI type } \\
\hline $\begin{array}{r}\text { UTI } \\
\text { sensitive to } \\
A B\end{array}$ & 36 & $4.7(3.5)$ & $\begin{array}{l}3.0 \\
(0.6)\end{array}$ & $3.1(3.3)$ & $3.5(3.5)$ & $3.7(3.6)$ & $\begin{array}{l}13.4 \\
(26.3)\end{array}$ & $\begin{array}{l}3.5 \\
(3.1)\end{array}$ \\
\hline $\begin{array}{r}\mathrm{UTI} \\
\text { resistant to } \\
A B \text { or no } \\
A B\end{array}$ & 7 & $5.0(2.7)$ & $\begin{array}{l}3.6 \\
(3.6)\end{array}$ & $3.4(2.1)$ & $3.4(2.5)$ & $3.0(2.9)$ & $6.3(4.0)$ & $\begin{array}{l}4.3 \\
(4.1)\end{array}$ \\
\hline $\begin{array}{r}\text { UTI } \\
\text { unknown } \\
\text { sensitivity }\end{array}$ & 26 & $3.4(1.8)$ & $\begin{array}{l}1.7 \\
(1.6)\end{array}$ & $1.7(1.2)$ & $2.4(1.7)$ & $2.0(1.9)$ & $5.4(2.2)$ & $\begin{array}{l}3.2 \\
(2.0)\end{array}$ \\
\hline $\begin{array}{r}\text { No } \\
\text { significant } \\
\text { bacterial } \\
\text { growth } \\
\text { (Urethral } \\
\text { syndrome) }\end{array}$ & 92 & $4.3(3.2)$ & $\begin{array}{l}1.6 \\
(2.1)\end{array}$ & $2.8(2.7)$ & $2.9(2.9)$ & $3.1(3.5)$ & $\begin{array}{l}12.0 \\
(23.2)\end{array}$ & $\begin{array}{l}4.1 \\
(3.5)\end{array}$ \\
\hline \multicolumn{9}{|l|}{ Antibiotic } \\
\hline Yes & 160 & $4.3(3.0)$ & $\begin{array}{l}2.0 \\
(2.5)\end{array}$ & $2.7(2.6)$ & $3.0(2.9)$ & $3.0(3.2)$ & $\begin{array}{l}10.3 \\
(20.4)\end{array}$ & $\begin{array}{l}3.8 \\
(3.2)\end{array}$ \\
\hline No & 18 & $3.4(3.5)$ & $\begin{array}{l}2.3 \\
(3.6)\end{array}$ & $2.2(2.6)$ & $2.9(3.5)$ & $2.2(3.6)$ & $\begin{array}{l}10.4 \\
(22.3)\end{array}$ & $\begin{array}{l}4.2 \\
(3.6)\end{array}$ \\
\hline
\end{tabular}




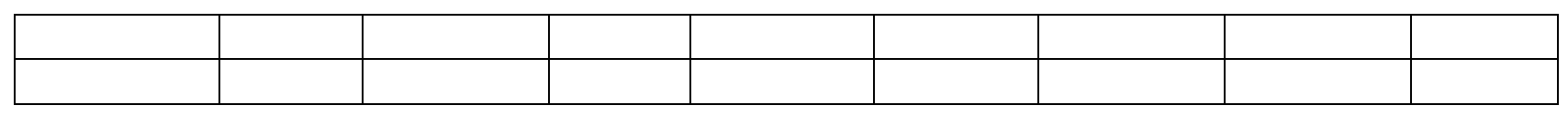


Table 2: AIA and health score

\begin{tabular}{|l|l|l|l|l|}
\hline & \multicolumn{3}{|l|}{ Day of consultation } & Day 4 \\
\hline AIA items and total score & Mean & SD & Mean & SD \\
\hline & 111 & & 96 & \\
\hline Cut down on time at work & 0.95 & 1.2 & 0.6 & 1.0 \\
\hline Accomplished less & 1.5 & 1.2 & 0.8 & 1.0 \\
\hline Limited in kind of work & 1.2 & 1.2 & 0.7 & 1.0 \\
\hline Difficulty performing work & 1.4 & 1.1 & 0.8 & 1.0 \\
\hline $\begin{array}{l}\text { Interfered with social } \\
\text { activity }\end{array}$ & 1.6 & 1.2 & 1.1 & 1.2 \\
\hline Total score & 6.6 & 4.9 & 4.1 & 4.6 \\
\hline & & & & \\
\hline Health score in general & 76 & 17 & 76 & 18 \\
\hline Health score today & 56 & 20 & 72 & 19 \\
\hline & & & & \\
\hline
\end{tabular}


Figure 1: Smartphone app

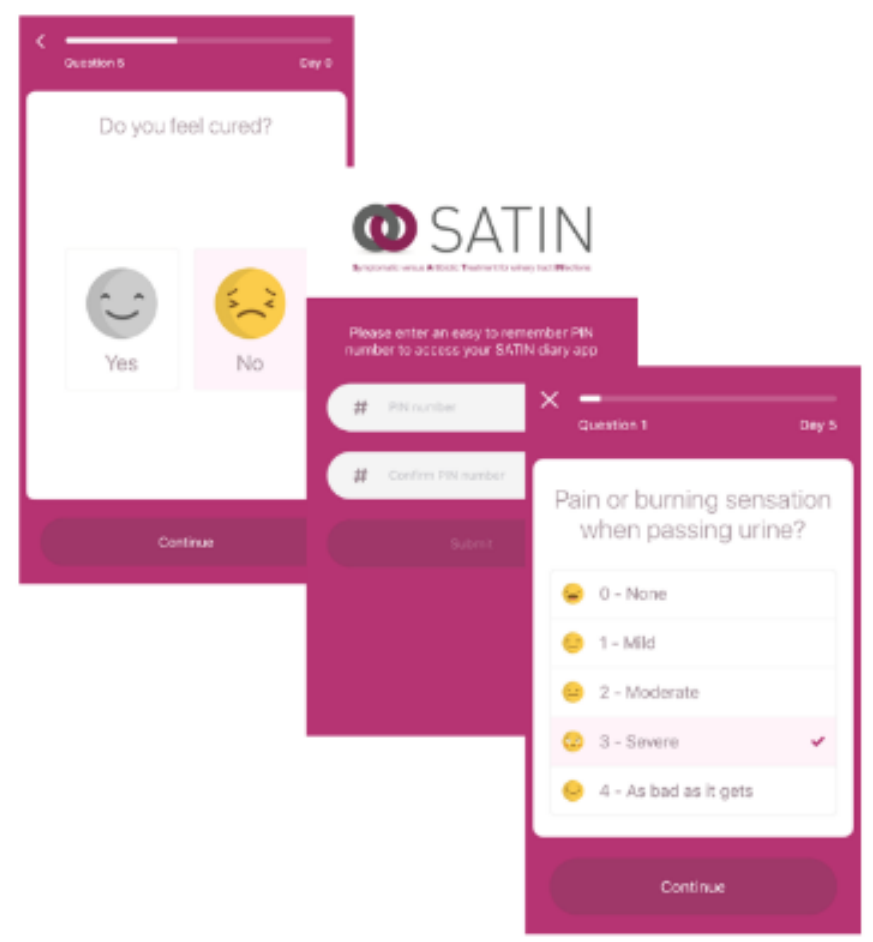


Figure 2: total symptom score by day

Day

$$
16
$$

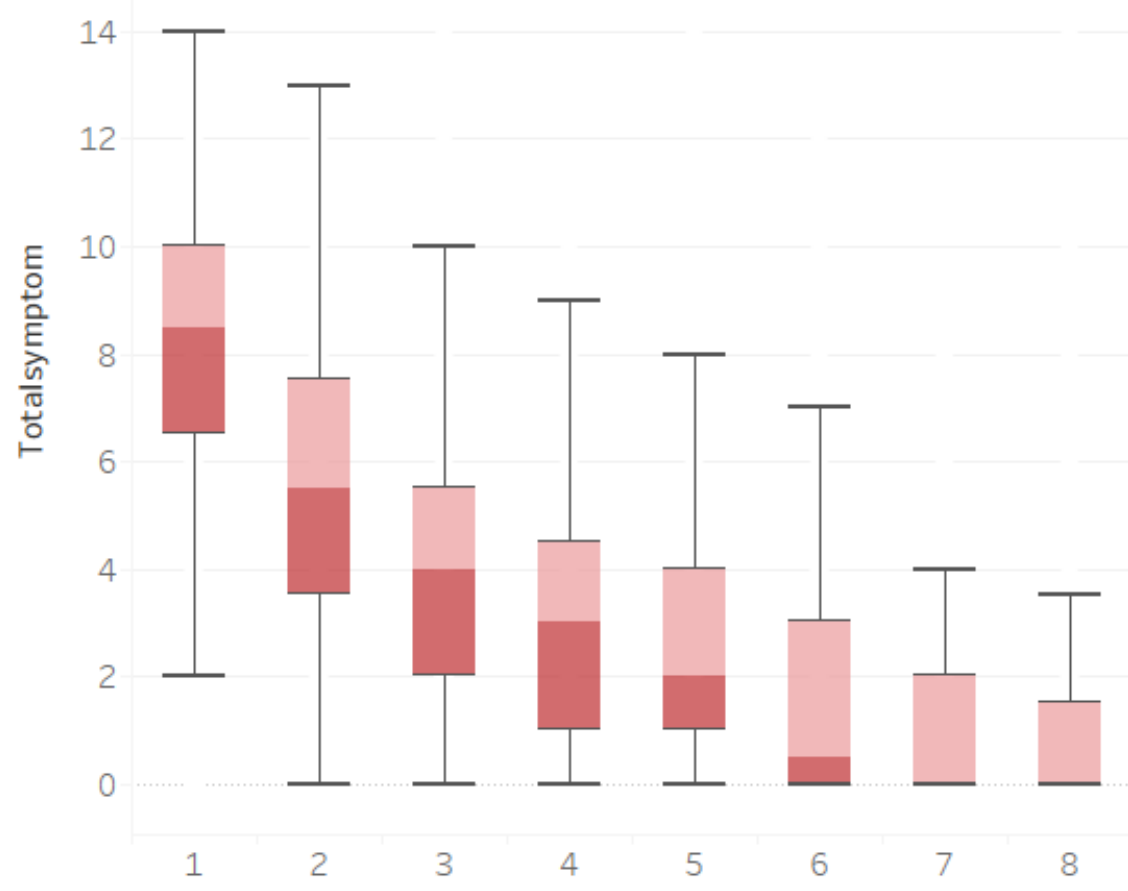


Figure 3: Mean duration of each symptom and total symptom scored moderate to severe for each symptom (dysuria, Frequency, urgency and lower abdominal pain), as well as any of these symptoms (average total duration).

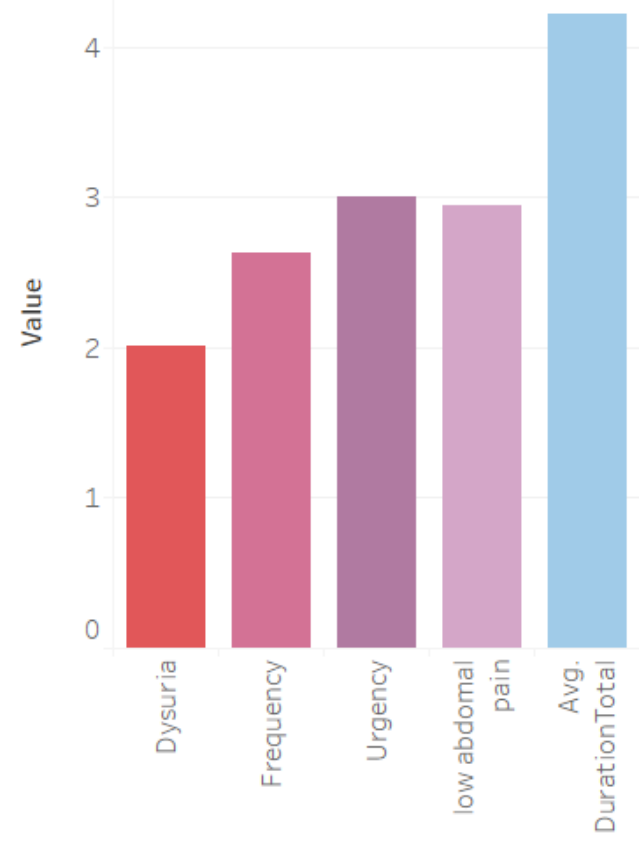


Figure 4: Day patient indicates to be cured from the day of consultation

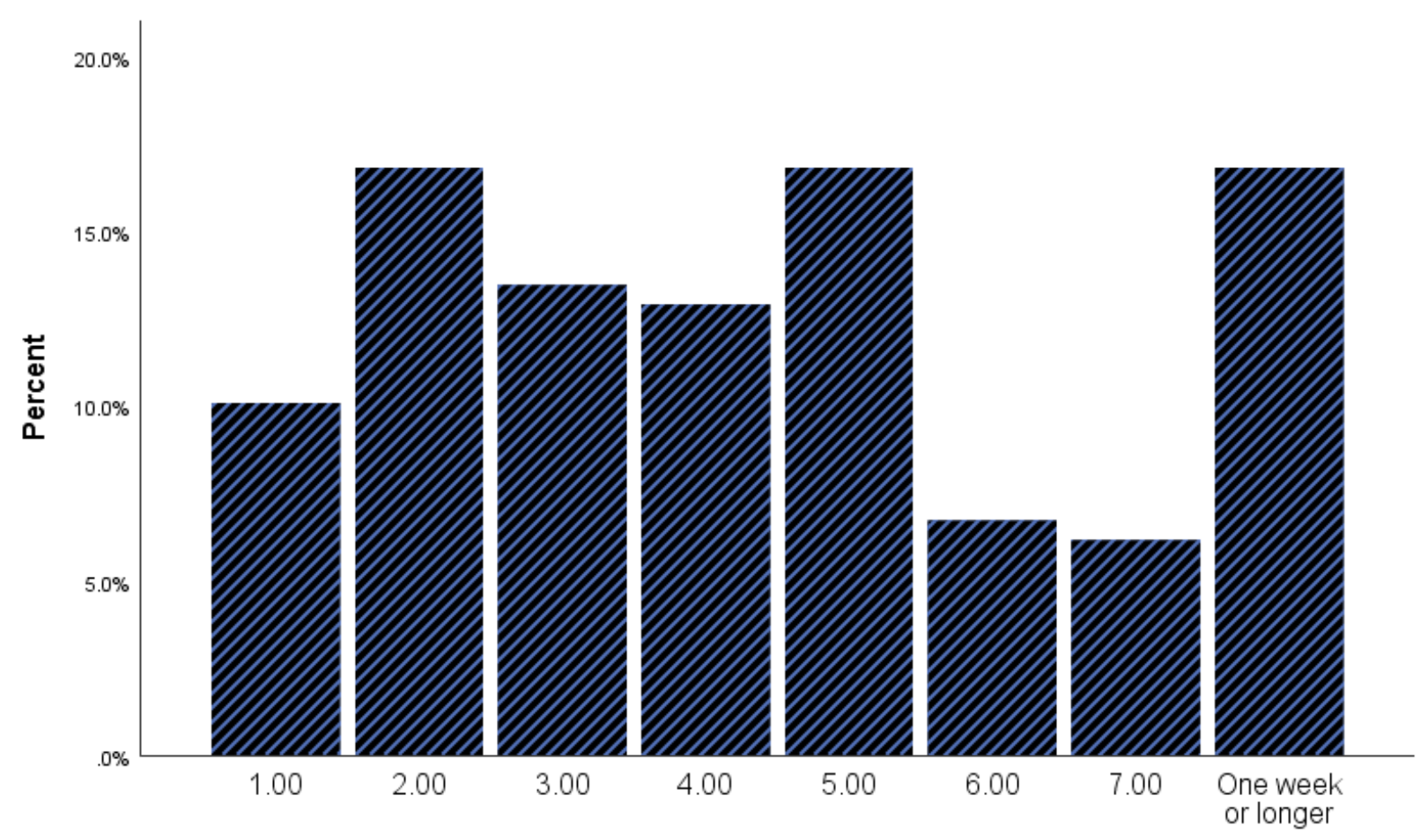

Day patient indicated to be cured 
Figure 5: Health score on the day and in general, on day 0 and day 5

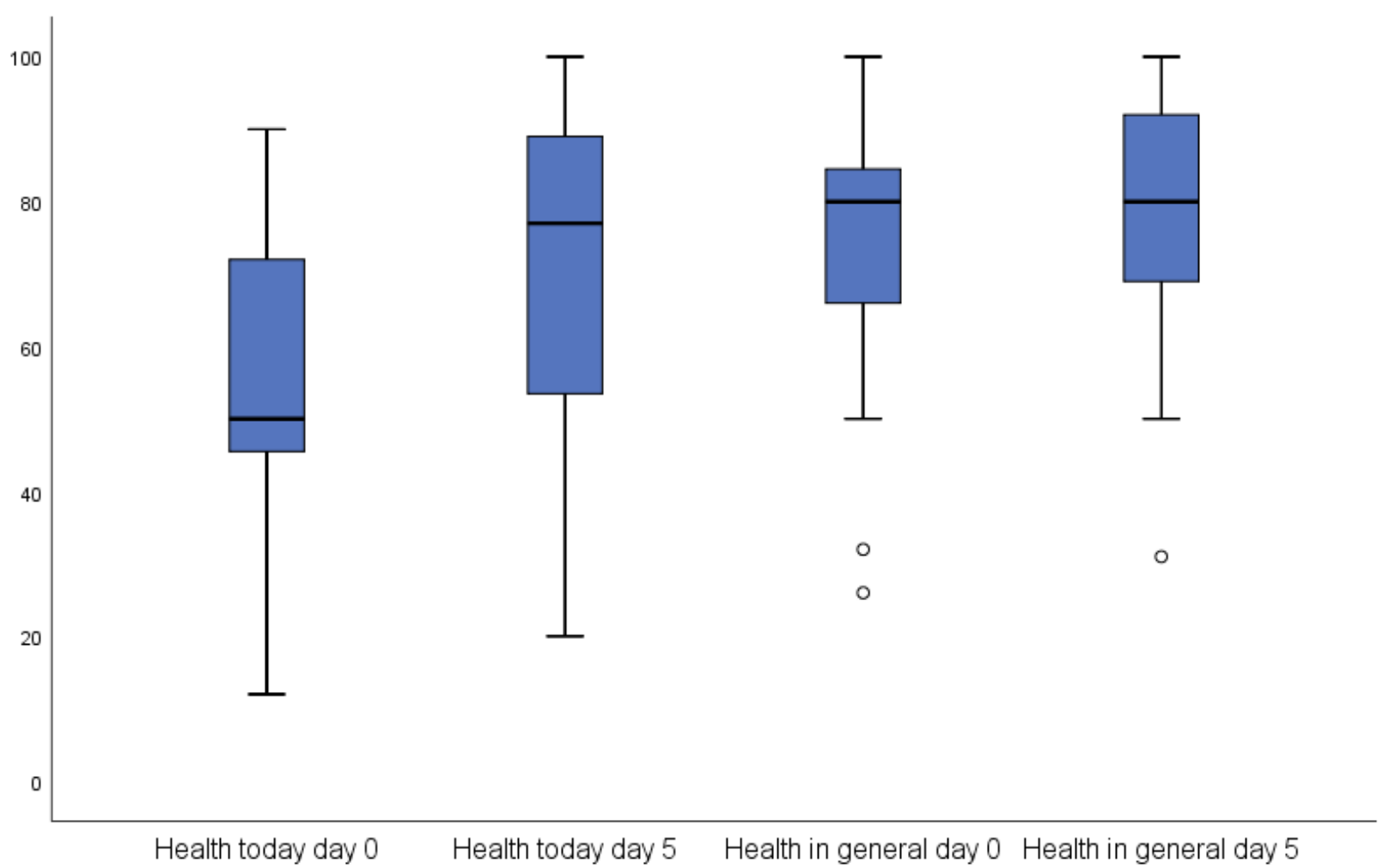




\section{References}

1. Foxman B. Epidemiology of Urinary Tract Infections: Incidence, Morbidity, and Economic Costs. Dis Mon. 2003;49.

2. Little P, Merriman R, Turner S, Rumsby K, Warner G, Lowes JA, et al. Presentation, pattern, and natural course of severe symptoms, and role of antibiotics and antibiotic resistance among patients presenting with suspected uncomplicated urinary tract infection in primary care: observational study. BMJ. 2010;340:b5633.

3. Gupta K, Hooton TM, Naber KG, Wullt Br, Colgan R, Miller LG, et al. International Clinical Practice Guidelines for the Treatment of Acute Uncomplicated Cystitis and Pyelonephritis in Women: A 2010 Update by the Infectious Diseases Society of America and the European Society for Microbiology and Infectious Diseases. Clinical Infectious Diseases. 2011;52(5):e103-e20.

4. Christiaens TC, De Meyere M, Verschraegen G, Peersman W, Heytens S, De Maeseneer JM. Randomised controlled trial of nitrofurantoin versus placebo in the treatment of uncomplicated urinary tract infection in adult women. Br J Gen Pract. 2002;52(482):729-34.

5. Little P, Moore MV, Turner S, Rumsby K, Warner G, Lowes JA, et al. Effectiveness of five different approaches in management of urinary tract infection: randomised controlled trial. BMJ. 2010;340:c199.

6. Hooton TM, Besser R, Foxman B, Fritsche TR, Nicolle LE. Acute uncomplicated cystitis in an era of increasing antibiotic resistance: a proposed approach to empirical therapy. Clin Infect Dis. 2004;39(1):75-80.

7. Vellinga A, Cormican M, Hanahoe B, Bennett K, Murphy AW. Antimicrobial management and appropriateness of treatment of urinary tract infection in general practice in Ireland. BMC Fam Pract. 2011;12(1):108.

8. Strategy for the control of Antimicrobial Resistance in Ireland (SARI). Management of infection guidance for primary care in Ireland 2008, updated 2014 [Available from: http://www.antibioticprescribing.ie/.

9. Vellinga A, Tansey S, Hanahoe B, Bennett K, Murphy AW, Cormican M. Trimethoprim and ciprofloxacin resistance and prescribing in urinary tract infection associated with Escherichia coli: a multilevel model. The Journal of antimicrobial chemotherapy. 2012.

10. Costelloe C, Metcalfe C, Lovering A, Mant D, Hay AD. Effect of antibiotic prescribing in primary care on antimicrobial resistance in individual patients: systematic review and meta-analysis. BMJ. 2010;340:c2096.

11. Coast J, Smith RD. Antimicrobial resistance: cost and containment. Expert review of antiinfective therapy. 2003;1(2):241-51.

12. Butler CC, Hood K, Verheij T, Little P, Melbye H, Nuttall J, et al. Variation in antibiotic prescribing and its impact on recovery in patients with acute cough in primary care: prospective study in 13 countries. BMJ. 2009;338:b2242.

13. Little P, National Co-ordinating Centre for HTA. Dipsticks and diagnostic algorithms in urinary tract infection : development and validation, randomised trial, economic analysis, observational cohort and qualitative study. Perth: Prepress Projects on behalf of NCCHTA; 2009. xi, 96 p. p.

14. Little P, Turner S, Rumsby K, Warner G, Moore M, Lowes JA, et al. Developing clinical rules to predict urinary tract infection in primary care settings: sensitivity and specificity of near patient tests (dipsticks) and clinical scores. Br J Gen Pract. 2006;56(529):606-12.

15. McNulty CA, Richards J, Livermore DM, Little P, Charlett A, Freeman E, et al. Clinical relevance of laboratory-reported antibiotic resistance in acute uncomplicated urinary tract infection in primary care. The Journal of antimicrobial chemotherapy. 2006;58(5):1000-8.

16. Gagyor I, Bleidorn J, Kochen MM, Schmiemann G, Wegscheider K, Hummers-Pradier E. Ibuprofen versus fosfomycin for uncomplicated urinary tract infection in women: randomised controlled trial. BMJ. 2015;351:h6544. 
17. Vik I, Bollestad M, Grude N, Baerheim A, Damsgaard E, Neumark T, et al. Ibuprofen versus pivmecillinam for uncomplicated urinary tract infection in women-A double-blind, randomized noninferiority trial. 2018;15(5):e1002569.

18. Butler CC, Francis N, Thomas-Jones E, Llor C, Bongard E, Moore M, et al. Variations in presentation, management, and patient outcomes of urinary tract infection: a prospective fourcountry primary care observational cohort study. British Journal of General Practice. 2017;67(665):e830-e41.

19. Holm A, Cordoba G, Møller Sørensen T, Rem Jessen L, Frimodt-M $\varnothing$ ller N, Siersma V, et al. Effect of point-of-care susceptibility testing in general practice on appropriate prescription of antibiotics for patients with uncomplicated urinary tract infection: a diagnostic randomised controlled trial. BMJ Open. 2017;7(10).

20. Stone AA, Shiffman S, Schwartz JE, Broderick JE, Hufford MR. Patient compliance with paper and electronic diaries. Control Clin Trials. 2003;24(2):182-99.

21. Litcher-Kelly L, Kellerman Q, Stone AA, Hanauer SB. Feasibility and utility of an electronic diary to assess self-report symptoms in patients with inflammatory bowel disease. Annals of Behavioral Medicine. 2007;33(2):207-12.

22. Quinn P, Goka J, Richardson H. Assessment of an electronic daily diary in patients with overactive bladder. BJU Int. 2003;91(7):647-52.

23. Kupczyk M, Haque S, Sterk PJ, Niżankowska-Mogilnicka E, Papi A, Bel EH, et al. Detection of exacerbations in asthma based on electronic diary data: results from the 1-year prospective BIOAIR study. Thorax. 2013;68(7):611-8.

24. Kay M, Santos J, Takane M. mHealth: new horizons for health through mobile technologies: second global survey on eHealth. In: eHealth WGOf, editor.: World Health Organisation; 2011.

25. Stinson JN, Petroz GC, Tait G, Feldman BM, Streiner D, McGrath PJ, et al. e-Ouch: usability testing of an electronic chronic pain diary for adolescents with arthritis. Clin J Pain. 2006;22(3):295305.

26. Kristjansdottir OB, Fors EA, Eide E, Finset A, Stensrud TL, van Dulmen S, et al. A smartphonebased intervention with diaries and therapist-feedback to reduce catastrophizing and increase functioning in women with chronic widespread pain: randomized controlled trial. J Med Internet Res. 2013;15(1):e5.

27. Marcano Belisario JS, Huckvale K, Greenfield G, Car J, Gunn LH. Smartphone and tablet self management apps for asthma. Cochrane Database Syst Rev. 2013(11):Cd010013.

28. Arsand E, Muzny M, Bradway M, Muzik J, Hartvigsen G. Performance of the first combined smartwatch and smartphone diabetes diary application study. Journal of diabetes science and technology. 2015;9(3):556-63.

29. Park JW, Chu MK, Kim JM, Park SG, Cho SJ. Analysis of Trigger Factors in Episodic Migraineurs Using a Smartphone Headache Diary Applications. PLoS One. 2016;11(2):e0149577.

30. Vellinga A, Galvin S, Duane S, Callan A, Bennett K, Cormican M, et al. Intervention to improve the quality of antimicrobial prescribing for urinary tract infection: a cluster randomized trial. CMAJ. 2016;188(2):108-15.

31. Duane S, Tandan M, Murphy AW, Vellinga A. Using Mobile Phones to Collect Patient Data: Lessons Learned From the SIMPle Study. JMIR Res Protoc. 2017;6(4):e61.

32. Vik I, Bollestad M, Grude N, Bærheim A, Mölstad S, Bjerrum L, et al. Ibuprofen versus mecillinam for uncomplicated cystitis - a randomized controlled trial study protocol. BMC Infectious Diseases. 2014;14(1):1-6.

33. Wild DJ, Clayson DJ, Keating K, Gondek K. Validation of a patient-administered questionnaire to measure the activity impairment experienced by women with uncomplicated urinary tract infection: the Activity Impairment Assessment (AIA). Health Qual Life Outcomes. 2005;3:42.

34. Greiner W, Weijnen T, Nieuwenhuizen M, Oppe S, Badia X, Busschbach J, et al. A single European currency for EQ-5D health states. Results from a six-country study. Eur J Health Econ. 2003;4(3):222-31. 
35. Duane S, Vellinga A, Murphy AW, Cormican M, Smyth A, Healy P, et al. COSUTI: a protocol for the development of a core outcome set (COS) for interventions for the treatment of uncomplicated urinary tract infection (UTI) in adults. Trials. 2019;20(1):106.

36. O'Connor N, Breen R, Carton M, Mc Grath I, Deasy N, Collins C, et al. Improving the quality of antibiotic prescribing through an educational intervention delivered through the out-of-hours general practice service in Ireland. European Journal of General Practice. 2020;26(1):119-25.

37. Wild DJ, Clayson DJ, Keating K, Gondek K. Validation of a patient-administered questionnaire to measure the activity impairment experienced by women with uncomplicated urinary tract infection: the Activity Impairment Assessment (AIA). Health Qual Life Outcomes. 2005;3:42-. 